\title{
Heterogeneity within AML with CEBPA mutations; only CEBPA double mutations, but not single CEBPA mutations are associated with favourable prognosis
}

\author{
T Pabst*, , M Eyholzer', J Fos² and BU Mueller² \\ 'Department of Medical Oncology and Clinical Research, University Hospital Bern and University Bern, Bern, Switzerland; '2Department of Internal \\ Medicine and Clinical Research, University Hospital Bern and University Bern, Bern, Switzerland
}

\begin{abstract}
CCAAT/enhancer binding protein alpha (CEBPA) mutations in AML are associated with favourable prognosis and are divided into $\mathrm{N}$ - and C-terminal mutations. The majority of AML patients have both types of mutations. We assessed the prognostic significance of single $(n=7)$ and double $(n=12)$ CEBPA mutations among 224 AML patients. Double CEBPA mutations conferred a decisively favourable overall $(P=0.006)$ and disease-free survival $(P=0.013)$. However, clinical outcome of patients with single CEBPA mutations was not different from CEBPA wild-type patients. In a multivariable analysis, only double - but not single - CEBPA mutations were identified as independent prognostic factors. These findings indicate heterogeneity within $A M L$ patients with CEBPA mutations.
\end{abstract}

British Journal of Cancer (2009) I 00, I343-1346. doi: I0.1038/sj.bjc.6604977 www.bjcancer.com

Published online 10 March 2009

(c) 2009 Cancer Research UK

Keywords: prognosis; AML; mutations; CEBPA; risk assessment

One of the crucial transcription factors for myeloid cell development is the CCAAT/enhancer binding protein alpha (CEBPA) (Rosenbauer and Tenen, 2007). Targeted disruption of CEBPA results in a selective block of granulocyte maturation (Zhang et al, 1997), whereas conditional expression of CEBPA in precursor cells is sufficient to trigger granulocytic differentiation (Radomska et al, 1998). In AML patients, deregulation of CEBPA function is a common event comprising of genomic mutations (Pabst et al, 2001a; Frohling et al, 2004; Nerlov, 2004), transcriptional and posttranscriptional suppression (Pabst et al, 2001b; Helbling et al, 2004; Helbling et al, 2005), and inactivation by phosphorylation (Radomska et al, 2006).

Two types of CEBPA mutations are predominantly seen: frameshift mutations in the $\mathrm{N}$-terminal truncate the wild-type protein, whereas formation of a dominant-negative $30-\mathrm{kDa}$ peptide initiated from an ATG further downstream is not affected. In contrast, $\mathrm{C}$-terminal CEBPA mutations are in-frame insertions or deletions, thereby affecting DNA binding and homo- or heterodimerisation with other CEBP family members (Nerlov, 2004). The majority of AML patients with CEBPA mutations have both types of mutations, usually on different alleles. However, both types can occur as single CEBPA mutations.

Earlier study has indicated that CEBPA mutations in cytogenetically normal AML patients are associated with favourable prognosis (Frohling et al, 2004; Bienz et al, 2005; Gaidzik and Dohner (2008); Marcucci et al, 2008; Schlenk et al, 2008). Here, we assessed the prognostic significance of the different types of CEBPA

* Correspondence: Professor T Pabst; E-mail: thomas.pabst@insel.ch Received 13 November 2008; revised 17 February 2009; accepted 18 February 2009; published online 10 March 2009 mutations among a cohort of 224 consecutive AML patients of all subtypes. By direct sequencing, we identified 12 patients with double and 7 with single heterozygous CEBPA mutations. Patients with double $C E B P A$ mutations represented the combination of $\mathrm{C}$ - and N-terminal mutation types. We found that favourable prognosis was exclusively associated with the double CEBPA mutation status, whereas the clinical outcome of patients with single $C E B P A$ mutations did not differ from $C E B P A$ wild-type patients.

\section{MATERIALS AND METHODS}

\section{Patients}

Malignant cells were collected at diagnosis from the Ficollseparated mononucleated cells of bone marrow aspirates (132 patients) or peripheral blood (92 patients) from consecutive AML patients seen at the Department of Oncology, University Hospital, Bern, Switzerland between 2001 and 2007. Informed consent from all patients was obtained according to the Declaration of Helsinki, and the studies were approved by decisions of the local ethics committee of Bern, Switzerland. Patients were uniformly treated within the HOVON/SAKK 30/00 protocol.

\section{CEBPA mutational analysis}

The entire coding region of the CEBPA gene was amplified using three overlapping PCR primer pairs as described earlier (Bienz et al, 2005). Sequences of the primers are listed in Supplementary Table S1. PCR products were sequenced in both directions. Abnormal sequencing results were repeated twice in both directions, including repetitions of PCR. 


\section{Reporter gene assays}

H1299 cells were transfected with $80 \mathrm{ng}$ of luciferase plasmid encoding an oligomeric CEBPA site, together with $20 \mathrm{ng}$ of pcDNA3-CEBPA or empty pcDNA3 vector along with $1.0 \mathrm{ng}$ of CMV-Renilla plasmid. After $24 \mathrm{~h}$, luciferase activities were determined (Pabst et al, 2001a). Each transfection experiment was repeated at least three times.

Protein isolation and western blot analyses: Fifty micrograms of extracted proteins from whole-cell lysates were analysed with a rabbit polyclonal antibody against CEBPA (1:1000; Santa Cruz Biotechnology, Santa Cruz, CA, USA.; sc-61).

\section{Statistical Analysis}

Patients alive without progression or relapse by the time of analysis were censored at the time of their last follow-up. Time-toevent curves were constructed according to the Kaplan-Meier method and were compared with the log-rank $\chi^{2}$-test.

\section{RESULTS AND DISCUSSION}

By sequencing the entire coding region, we identified 19 patients with CEBPA mutations in our cohort of $224 \mathrm{AML}$ patients at diagnosis. Of these 12 patients had the combination of the $\mathrm{N}$ - and the C-terminal type of mutation, further referred here as double $C E B P A$ mutation, and 7 patients had a single CEBPA mutation. Patients with the in-frame insertion polymorphism in the second transactivation domain (Wouters et al, 2007), with base pair variation(s) that did not lead to amino acid changes, or with inframe sequence variations of unknown significance (one patient), were not considered in the further analysis. All CEBPA mutations are presented in detail in Supplementary Tables S2A and S2B. Two patients with a single point mutation encoding a novel stop codon located downstream of the alternative ATG at position 120 were not of the classic $\mathrm{N}$-terminal mutation type, as formation of the $30-\mathrm{kDa}$ peptide was also affected, but they were classified as having single CEBPA mutations because of the functional relevance of these particular mutations.

We identified CEBPA mutations exclusively in patients below the age of 61 years at diagnosis. Thus, only AML patients in this age range were studied in the control group of $C E B P A$ wild-type patients, allowing comparison of patients treated within the same protocol. We detected no differences between patients with single and double CEBPA mutations with regard to FAB subtypes, leucocytes at diagnosis, percentage of peripheral blasts at diagnosis, bone marrow infiltration at diagnosis, and LDH levels (Supplementary Table S3).
A 100

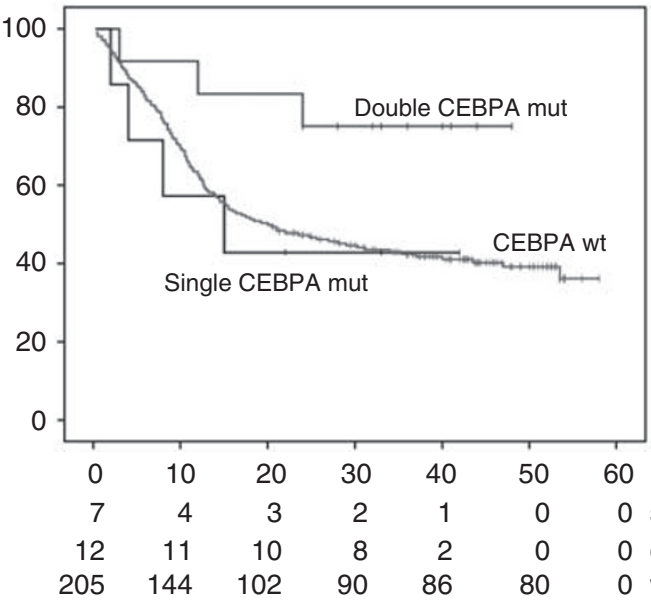

C

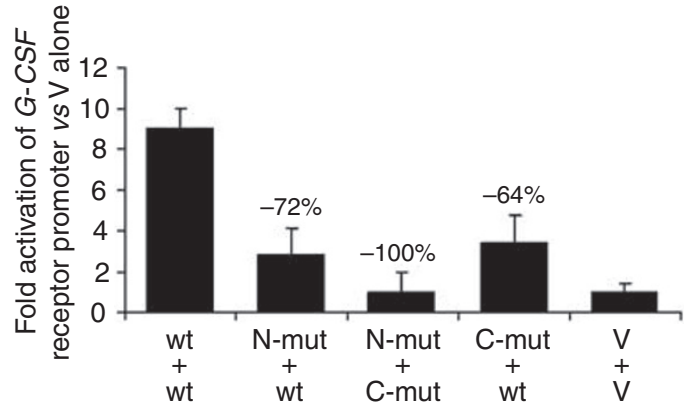

B

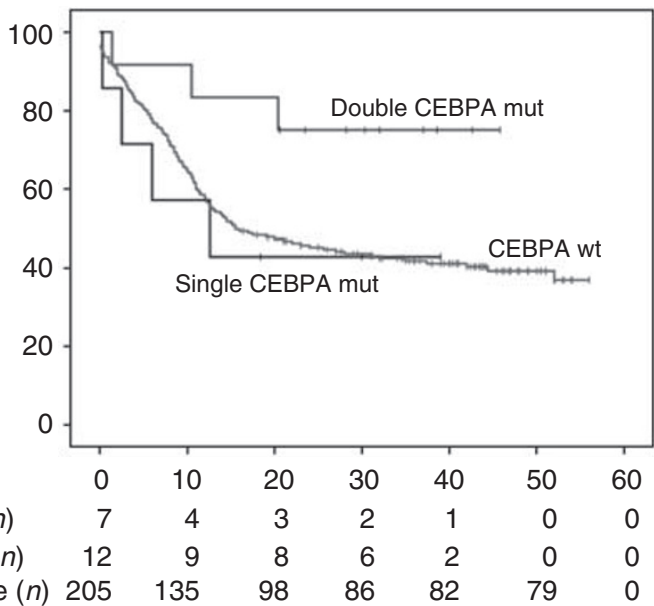

D

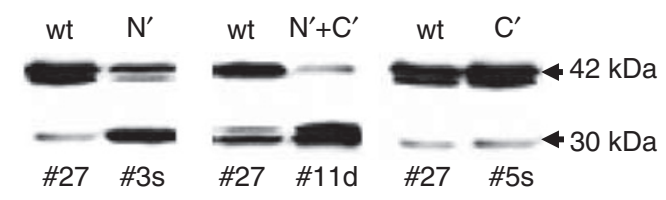

E

\begin{tabular}{|lllll|}
70 & 97 & $127 \quad 200$ & 278 & 358 \\
\hline & & & \\
\hline$\rightarrow 42 \mathrm{kDa}$ & $\square 30 \mathrm{kDa}$ & \\
\hline & & & \\
\hline
\end{tabular}

Figure I (A) Overall survival of AML patients without CEBPA mutations ( $w t ; n=205)$, with a single $(n=7)$, and with the combination of $C$ - and $N$-terminal CEBPA mutations (double; $n=12$ ). Patients who are alive were censored at the last follow-up. $X$-axis indicates months, $Y$-axis is probability of survival. (B) Disease-free survival of AML patients without ( $w t ; n=205)$ ), with a single $(n=7)$, or with double $(n=12)$ CEBPA mutations. (C) Transient transfection experiments in HI299 cells using equal amounts of pcDNA3 expression plasmids encoding human CEBPA wild-type (wt), the N-terminal frameshift mutation 245delC (as present in patient \#3s in Supplementary Table S2), the C-terminal in-frame mutation I079- I080ins TCT (as present in patient \#5s), and the combination of both plasmids. V: pcDNA3 expression plasmid alone. The luciferase reporter construct encodes an oligomeric CEBPA binding site. (D) Western blot analyses for CEBPA protein using whole-cell lysates of patients \#27 (AML-MI with a normal karyotype and no abnormalities in CEBPA, FLT3, and NPMI), \#3s (AML-M2 with the N-terminal frame-shift mutation 245delC), \#I Id (AML-MI with both the N-terminal 2I3insAG and the C-terminal 1088-1089insCCG mutations), and \#5 s (AML-MI with the C-terminal in-frame mutation I079- I080insTCT). (E) Schematic presentation of CEBPA wild-type protein (upper panel) and the 30-kDa peptide initiated at the ATG at amino acid I20 (lower panel). Black bars indicate the two transactivation domains, and grey bars represent the region for DNA binding and homo-/heterodimerisation. 
Table I Multivariable analysis for overall survival and disease-free survival to assess the prognostic significance of single vs double CEBPA mutations in AML patients.

\begin{tabular}{|c|c|c|c|c|}
\hline & Overall survival hazard ratio $(95 \% \mathrm{Cl})$ & $P$-value & Disease-free survival hazard ratio $(95 \% \mathrm{Cl})$ & $P$-value \\
\hline CEBPA double ${ }^{a}$ & $0.28(0.16-0.58)$ & $<0.00$ l* & $0.30(0.18-0.60)$ & $<0.001 *$ \\
\hline NPMI ${ }^{\mathrm{b}}$ & $0.56(0.43-0.75)$ & $<0.00 I^{*}$ & $0.54(0.41-0.74)$ & $<0.001^{*}$ \\
\hline WBC ${ }^{d}$ & $1.38(0.99-1.79)$ & $0.022 *$ & $1.35(0.98-1.74)$ & $0.036 *$ \\
\hline $\mathrm{Age}^{\mathrm{e}}$ & $1.15(1.06-1.26)$ & $0.008 *$ & $1.12(1.02-1.18)$ & $0.014 *$ \\
\hline
\end{tabular}

Abbreviations: $\mathrm{Cl}=$ confidence interval; NPMI = nucleophosmin; FLT3=fms-related tyrosine kinase 3; ITD = internal tandem duplication; WBC = white blood cell count;

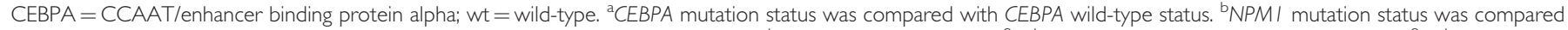

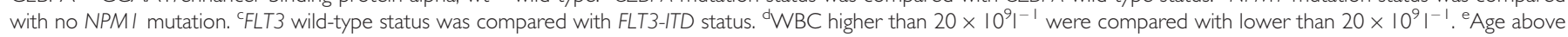
50 (to 60) years was compared with age below 50 years. * $P$-values $<0.05$ were considered significant.

Remarkably, additional molecular abnormalities were exclusively detected in the single CEBPA mutation group, including one patient with FLT3-ITD and one with NPM1 mutation. All patients with CEBPA mutations had a normal karyotype with the exception of one patient with monosomy 7 in the single CEBPA mutation group and one with del6q24 in the double CEBPA mutation group. Results of karyotype and molecular analyses are given in Supplementary Table S4.

Comparing patients with single vs double CEBPA mutations, we found that the clinical outcome differed markedly (Supplementary Table S5). A complete remission after induction chemotherapy was achieved in all the 19 AML patients with CEBPA mutations. However, patients with single $C E B P A$ mutations had a significantly worse median overall survival (OS) of 15 months (Figure 1A; $P=0.006$ ) and disease-free survival (DFS) of 12 months (Figure 1B; $P=0.013$ ) compared with patients with double CEBPA mutations in whom both median DFS and OS were not reached after a median follow-up of 34 months. Moreover, the course of the disease of patients with single CEBPA mutations was not different both for OS and DFS from CEBPA wild-type patients. Finally, in a multivariable analysis discriminating white blood cell count, age, NPM1 mutations, and FLT3-ITD, only double CEBPA mutations, but not single $C E B P A$ mutations, were identified as independent prognostic factors (Table 1). The CEBPA mutation status per se turned out to be of independent prognostic significance.

To illustrate the consequences of single vs double CEBPA mutations in terms of CEBPA function, we transfected H1299 cells with expression plasmids encoding $C E B P A$ wild-type, an N-terminal frame-shift mutation, a C-terminal in-frame mutation, or the combination of both plasmids, and we determined the potential of these CEBPA peptides to activate a target promoter sequence as present in the G-CSF receptor promoter. As illustrated in Figure 1C, both the $\mathrm{N}$ - and the C-terminal CEBPA mutant peptides inhibited wild-type $C E B P A$ protein in a dominant-negative manner, by reducing its activation potential by roughly $70 \%$. However, it is

\section{REFERENCES}

Bienz M, Ludwig M, Oppliger E, Mueller BU, Ratschiller D, Solenthaler M, Fey MF, Pabst T (2005) Risk assessment in patients with acute myeloid leukemia and a normal karyotype. Clin Cancer Res 11: 1416-1425

Frohling S, Schlenk RF, Stolze I, Bihlmayr J, Benner A, Kreitmeier S, Tobis K, Döhner H, Döhner K (2004) CEBPA mutations in younger adults with acute myeloid leukemia and normal cytogenetics: prognostic relevance and analysis of cooperating mutations. J Clin Oncol 22: 624-633

Gaidzik V, Dohner K (2008) Prognostic implications of gene mutations in acute myeloid leukemia with normal cytogenetics. Semin Oncol 35: 346-355 Helbling D, Mueller BU, Timchenko NA, Hagemeijer A, Jotterand M, Meyer-Monard S, Lister A, Rowley JD, Huegli B, Fey MF, Pabst T (2004) The leukemic fusion gene AML1-MDS1-EVI1 suppresses CEBPA in acute important that the combination of both mutant constructs - in the absence of wild-type protein - failed to activate at all. This suggests that $C E B P A$ activity is completely abolished in malignant cells of patients with double CEBPA mutations, whereas it is retained to some degree in patients with single $C E B P A$ mutations. In addition, Figure $1 \mathrm{D}$ presents the various $C E B P A$ peptides made in malignant cells from AML patients with different types of $C E B P A$ mutations. It indicates that $\mathrm{N}$-terminal frame-shift CEBPA mutations, in fact, decisively decrease the amount of wild-type $C E B P A$ protein, whereas the $30-\mathrm{kDa}$ peptide is detectable at a higher amount.

In conclusion, our findings indicate that there is relevant prognostic heterogeneity within AML patients with CEBPA mutations. Double $C E B P A$ mutations are associated with distinctly favourable prognosis, whereas clinical outcome of AML patients with single CEBPA mutations is not different from CEBPA wildtype patients. However, the number of patients with a single CEBPA mutation is limited in our collection of patients, and larger series are needed to definitively assess its prognostic significance.

\section{ACKNOWLEDGEMENTS}

We thank Barbara Huegli and Deborah Shan for help with sample preparation. TP designed and performed the research, and wrote the paper; ME performed the research; JF analysed the data; and BUM designed the research and wrote the paper. This work was supported by grants from the Swiss National Science Foundation SF $310000-109388$ (to TP) and SF 310000-113761 (to BUM).

\section{Conflict of interest}

The authors declare no conflict of interests.

Supplementary Information accompanies the paper on British Journal of Cancer website (http://www.nature.com/bjc)

myeloid leukemia by activation of Calreticulin. Proc Natl Acad Sci USA 101: $13312-13317$

Helbling D, Mueller BU, Timchenko NA, Schardt J, Eyer M, Betts DR, Jotterand M, Meyer-Monard S, Fey MF, Pabst T (2005) CBFB-SMMHC is correlated with increased calreticulin expression and suppresses the granulocytic differentiation factor CEBPA in AML with inv(16). Blood 106: $1369-1375$

Marcucci G, Maharry K, Radmacher MD, Mrozek K, Vukosavljevic T, Paschka P, Whitman SP, Langer C, Baldus CD, Liu CG, Ruppert AS, Powell BL, Carroll AJ, Caligiuri MA, Kolitz JE, Larson RA, Bloomfield CD (2008) Prognostic significance of, and gene and microRNA expression signatures associated with, CEBPA mutations in cytogenetically normal 
acute myeloid leukemia with high-risk molecular features: a cancer and leukemia group B study. J Clin Oncol 26: 5078-5087

Nerlov C (2004) C/EBP alpha mutations in acute myeloid leukaemias. Nat Rev Cancer 4: 394-400

Pabst T, Mueller BU, Zhang P, Radomska HS, Narravula S, Schnittger S, Behre G, Hiddemann W, Tenen DG (2001a) Dominant-negative mutations of CEBPA, encoding CCAAT/enhancer binding protein-alpha (C/EBPalpha), in acute myeloid leukemia. Nat Genet 27: 263-270

Pabst T, Mueller BU, Harakawa N, Schoch C, Haferlach T, Behre G, Hiddemann W, Zhang DE, Tenen DG (2001b) AML1-ETO downregulates the granulocytic differentiation factor C/EBPalpha in $t(8 ; 21)$ myeloid leukemia. Nat Med 7: 444-451

Radomska HS, Huettner CS, Zhang P, Cheng T, Scadden DT, Tenen DG (1998) CCAAT/enhancer binding protein alpha is a regulatory switch sufficient for induction of granulocytic development from bipotential myeloid progenitors. Mol Cell Biol 18: 4301-4314

Radomska HS, Basseres DS, Zheng R, Zhang P, Dayaram T, Yamamoto Y, Sternberg DW, Lokker N, Giese NA, Bohlander SK, Schnittger S, Delmotte
MH, Davis RJ, Small D, Hiddemann W, Gilliland DG, Tenen DG (2006) Block of C/EBP alpha function by phosphorylation in acute myeloid leukemia with FLT3 activating mutations. J Exp Med 203: 371 -381

Rosenbauer F, Tenen DG (2007) Transcription factors in myeloid development: balancing differentiation with transformation. Nat Rev Immunol 7: 105-117

Schlenk RF, Dohner K, Krauter J, Frohling S, Corbacioglu A, Bullinger L, Habdank M, Spath D, Morgan M, Benner A, Schlegelberger B, Heil G, Ganser A, Dohner H (2008) Mutations and treatment outcome in cytogenetically normal acute myeloid leukemia. N Engl J Med 358: 1909-1918

Wouters BJ, Louwers I, Valk PJ, Lowenberg B, Delwel R (2007) A recurrent in-frame insertion in a CEBPA transactivation domain is a polymorphism rather than a mutation that does not affect gene expression profiling-based clustering of AML. Blood 109: 389-390

Zhang DE, Zhang P, Wang ND, Hetherington CJ, Darlington GJ, Tenen DG (1997) Absence of granulocyte colony-stimulating factor signaling and neutrophil development in CCAAT enhancer binding protein alphadeficient mice. Proc Natl Acad Sci U S A 94: 569-574 